\begin{tabular}{lllllllllllllllllllllllllllllllll}
\hline$R$ & $E$ & $V$ & I & S & T & A & D & E & E & S & T & U & D & I & O & S & I & N & T & E & R & N & A & C & I & O & N & A & L & E & S
\end{tabular}

\title{
Japón y Corea del Sur: relaciones bilaterales y seguridad regional
}

\author{
Cristián Maldonado M.
}

\begin{abstract}
Numerosos autores han vaticinado que el siglo XXI será considerado como el «Siglo del Pacífico», en directa referencia a las posibilidades de que esta extensa región llegue a transformarse en motor de la actividad económica y de la política mundial. Dentro de este contexto, puede afirmarse que por su influencia en la seguridad regional, los países del Noreste Asiático, particularmente, China, Japón, Corea del Sur y Corea del Norte, desempeñan uno de los papeles más importantes dentro de la Cuenca del Pacífico. Pese a ciertos periodos críticos, la post Guerra Fría ha permitido profundizar el acercamiento y la cooperación entre estos actores, especialmente, entre Japón y Corea del Sur, lo cual ha potenciado el paulatino surgimiento de un régimen de seguridad entre ambos países.
\end{abstract}

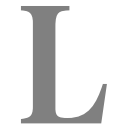

a siguiente investigación busca establecer que los actuales procesos de profundización de vínculos políticos y económicos entre Japón y Corea del Sur no solo favorece a la seguridad de ambos países sino también al subsistema regional del noreste de Asia, y al sistema global. En ese sentido, recurriendo a conceptos realistas, constructivistas y liberales, se pretende demostrar, que pese a las tensiones existentes y a una marcada contraposición de percepciones históricas, actualmente Japón y Corea del Sur se encuentran en un creciente proce- so de acercamiento en materia de seguridad, que ha permitido en gran parte superar la inestabilidad regional. A su vez, los vínculos entre ellos se basan en una milenaria relación entre Japón y la península, que ha tenido pronunciados altibajos, pero no se ha interrumpido; y reflejan, en un ámbito más localizado, la evolución histórica del sistema internacional.

En términos específicos, es posible manifestar que el grado de acercamiento o de conflictividad entre Japón y la península coreana influye directamente en el grado de estabilidad o de inestabilidad del 
noreste de Asia, planteamiento básico que da pie para formular la siguiente hipótesis: el acercamiento en materia de seguridad entre Japón y Corea del Sur ha sido producto de sus respectivas alianzas bilaterales con los Estados Unidos y de la amenaza compartida que representa Corea del Norte para cada uno de ellos. Desde el fin de la Guerra Fría, este diálogo de seguridad ha permitido profundizar los vínculos políticos y económicos, y atenuar el conflicto histórico (el legado colonial, primordialmente) entre Corea del Sur y Japón, favoreciendo la emergencia de un subsistema de seguridad regional que ha contribuido finalmente a mantener los equilibrios estratégicos en el noreste de Asia.

\section{No obstante altibajos, la milenaria relación entre Japón y la península coreana no se ha interrumpido.}

Cabe mencionar que si bien la profundización de los vínculos políticos $\mathrm{y}$ económicos constituye una variable dependiente del diálogo de seguridad, en la medida en que ese proceso avanza, se ha ido transformando, en una causa adicional de dicho diálogo, tanto por sí misma como por su influencia en la atenuación del conflicto histórico entre ambas partes.

\section{HACIA UNA COMUNIDAD DE SEGURIDAD}

De acuerdo con la teoría de Barry Buzan sobre los Complejos de Seguridad
Regional, puede sostenerse que la relación sistémica entre Corea del Sur y Japón se ha mantenido en el tiempo, porque no obstante su inestabilidad en ciertos periodos históricos, se basa en la capacidad de interacción. A su vez, esta capacidad de interacción puede estructurarse de acuerdo con lo que este mismo autor denomina «patrones durables de amistad o de enemistad». Según la definición original de Buzan, los Complejos de Seguridad Regional son «grupos de Estados cuyas preocupaciones fundamentales de seguridad los vinculan de manera suficientemente cercana, de tal modo que la seguridad nacional de cada uno no puede razonablemente considerarse en forma independiente de la de los demás» ${ }^{1}$. El sistema Corea del Sur-Japón responde adecuadamente a esta definición. Al mismo tiempo, dentro de cada complejo, la interdependencia en seguridad puede ser negativa, a través de la formación de conflictos, o positiva, es decir, progresar hacia un régimen o comunidad de seguridad. El sistema Corea del Sur-Japón ha evolucionado por ambos tipos de lógica. La forma más común de interacción ha sido la rivalidad o la búsqueda de alguna forma de equilibrio de poder, producto de la estructura anárquica de todo complejo. Sin embargo, una vez finalizada la Segunda Guerra Mundial, en los Estados Unidos, la dependencia mutua de ambas partes en materia de seguridad introdujo una transformación interna que en el actual período de post Guerra Fría ha permitido echar las bases de un incipiente régimen de seguridad.

Buzan, B. y Waever, O., Regions and Powers, Cambridge, Cambridge University Press, 2003, pp. 44. 


\section{Las nuevas dinámicas de interacción han permitido transformar las identidades nacionales.}

Por otro lado, y desde un punto de vista constructivista, las nuevas dinámicas de interacción generadas dentro del sistema Corea del Sur-Japón a partir del fin de la Segunda Guerra Mundial, también han posibilitado una paulatina transformación de las respectivas identidades nacionales, permitiendo el paso de una lógica de oposición a una de cooperación. Ello ha sido potenciado no sólo por la dependencia de ambos de los Estados Unidos sino que, particularmente desde el fin de la Guerra Fría, por la similitud de los principios que subyacen sus sistemas político y económico: la democracia y el libre mercado. De ese modo, los vínculos entre ambos han entrado a multiplicarse, e igual cosa ha sucedido con los agentes que protagonizan esas interacciones, más allá de las respectivas esferas estatales. Desde una perspectiva liberal, los costos de un conflicto entre Japón y Corea del Sur resultan muy superiores a los beneficios de solucionarlos por vía de la negociación, asunto que de cuando en cuando refleja su plausibilidad en el caso específico y no resuelto del disputado islote Tokdo (en coreano) o Takeshima (en japonés).

\section{RELACIONES POLÍTICO-ECONÓMICAS POST GUERRA FríA}

La desintegración de la Unión Soviética implicó el fin de la estructura bipolar del sistema internacional, alterando las dinámicas regionales en el noreste de Asia. Unido a la apertura de China, lo anterior significó la supresión de la amenaza comunista y su reemplazo por un deseo compartido de mantener la estabilidad regional. Sin embargo, en un principio las relaciones de seguridad entre Japón y Corea del Sur se mantuvieron dentro del esquema acostumbrado debido a la persistencia del régimen de Kim Il Sung en Corea del Norte. De ese modo, ambos países juzgaron necesario continuar sus alianzas bilaterales con los Estados Unidos, en el entendido de que tales mecanismos habían contribuido a evitar las confrontaciones en el noreste de Asia, y en la península coreana en particular. No obstante, la primacía de esta visión de balance realista no impidió que más tarde se desplegaran importantes esfuerzos de cooperación en el campo político y económico, que han influido en el desarrollo del diálogo de seguridad entre ambos. Por otro lado, no hay que menospreciar el impacto que provocan las diferentes interpretaciones sobre el pasado reciente, al punto de que en cierto sentido condicionan aquel diálogo y las relaciones bilaterales en general.

Durante los primeros años de la post Guerra Fría, los vínculos políticos y económicos entre Japón y Corea del Sur no experimentaron cambios significativos. Incluso tendieron a resentirse debido al acercamiento paralelo que el gobierno ja- 
ponés inició con Corea del Norte. Para el entonces presidente surcoreano, Roh Tae Woo, la actitud japonesa indicaba un posible trato preferencial hacia Corea del Norte, que amenazaba con incluir compensaciones de guerra superiores a las que Corea del Sur había logrado con Japón. No obstante, las sospechas respecto del desarrollo de armas nucleares en el Norte llevaron a que este último país tomara en cuenta las preocupaciones del Sur, comprometiéndose a no normalizar relaciones con el primero sin considerar las opiniones del segundo, política que el MOFA (Ministerio de Asuntos Exteriores de Japón) denominó renkei ${ }^{2}$.

La llegada de Kim Young Sam al poder, en 1993, puso a Corea del Sur en el camino del mercado global y de la democracia liberal. El primer presidente sin pasado militar desde 1960 dio inicio a una «nueva diplomacia», en donde «la democracia, la libertad y los derechos humanos serían componentes esenciales de su política exterior» ${ }^{3}$. En cambio, Japón, en medio de una fuerte recesión económica, tuvo que enfrentar por primera vez una severa inestabilidad política, ejemplificada por la pérdida de las elecciones de 1993 por el partido liberal demócrata.

La crisis nuclear desatada por Corea del Norte en 1994 puso a ambos países en la necesidad de profundizar sus vínculos. Desde esa fecha, Japón ha expresado un fuerte apoyo a Corea del Sur, incrementando el número de encuentros bilaterales, aunque dentro del patrón triangular de seguridad establecido con los Estados Unidos En una visita realizada a Tokio en 1995, el presidente Kim Young Sam expresó interés por establecer una relación bilateral «orientada hacia el futuro», que no estuviera solo concentrada en temas de seguridad. Al mismo tiempo, agregó la necesidad de que las relaciones económicas entre ambos apliquen una lógica económica y no política, en alusión a la «influencia emocional» que ejercen las diferencias sobre el pasado reciente ${ }^{4}$.

\section{La delimitación de la ZEE de la isla Tokdo/Takeshima reavivó las dificultades para superar el legado colonial.}

Las dificultades para superar el legado colonial volvieron a la superficie con motivo de la delimitación de las 200 millas de Zona Económica Exclusiva en torno a la disputada isla Tokdo/Takeshima, en 1996. Entre manifestaciones nacionalistas de todo tipo, se llegó al extremo de acusar a Japón de estar preparando incluso una especie de invasión. Sin embargo, la trascendencia del incidente pasó a segundo plano debido a que se produjo antes de una elección local en Corea del Sur, que puso de manifiesto una suerte de utilización política de la controversia.

\section{Ibidem.}

3 Chae-Jin Lee, «South Korean Foreign Relations face the Globalization Challenges», en Samuel S. Kim (ed.), Korea's Globalization, Cambridge, Cambridge University Press, 2000, p. 175.

4 Euichul Choi, «The ROK-Japan Relationship: A Korean Reappraisal», disponible en <www.nira.go.jp/ publ/review/95summer>. 


\section{Para manejar los problemas de liquidez de corto plazo se propuso crear un Fondo Monetario Asiático.}

El estallido de la crisis financiera a fines de 1997 impactó de diferente manera a ambos países. La onda expansiva llegó a Corea del Sur en noviembre, en medio de la debacle generalizada del sudeste asiático. La crisis sorprendió a este país en un momento de extrema fragilidad, con tasas astronómicas de endeudamiento, dentro de un ineficiente y distorsionado sistema financiero ${ }^{5}$. El FMI decidió intervenir con paquetes de ayuda para cada uno de los países afectados, a los cuales en un principio se sumó Japón, que estaba sumido en una corriente recesiva. Sin embargo, dado su alto nivel de relaciones económicas con los países afectados, decidió encarar la crisis con mayor profundidad. En septiembre de 1997, el ministro de finanzas, Mitsuzuka Hiroshi, propuso destinar 100 mil millones de dólares a la creación de un Fondo Monetario Asiático con el fin de manejar los problemas de liquidez a corto plazo. La idea encontró fuerte apoyo, pero también fuerte oposición. La principal crítica provino de los Estados Unidos, país que juzgó la propuesta como la virtual pérdida de su dominio económico en la región ${ }^{6}$. La propuesta concluyó por diluirse y Japón se plegó nuevamente a los lineamientos generales es- tipulados por el FMI, destinando, entre otros paquetes de rescate, 10 mil millones de dólares a Corea del Sur, y asignado 30 mil millones más como apoyo financiero para el Asia, medida que se conoció como el «Plan Miyazawa».

Las elecciones presidenciales de fines de 1997 en Corea del Sur dieron como ganador al veterano líder político, Kim Dae Jung, en pleno desarrollo de la crisis económica. El nuevo gobierno marcó un verdadero punto de inflexión en las relaciones entre Corea del Sur y Japón. En primer lugar, como parte de un riguroso ajuste interno, Corea abrió completamente sus mercados a los productos japoneses y liberalizó las medidas para la introducción de inversiones extranjeras. Dentro de este contexto, y bajo el paraguas de lo que él mismo denominó la sunshine policy, el presidente Kim Dae Jung efectuó una visita de Estado a Japón en octubre de 1998, que finalizó con la firma de una «Declaración conjunta sobre una nueva asociación para el siglo 21». El presidente Kim Dae Jung y el primer ministro japonés de ese período, Keizo Obuchi, acordaron promover y mejorar los vínculos entre ambos países, incluyendo la disposición a resolver las controversias sobre el pasado colonial japonés, así como el aumento de la cooperación política y de seguridad respecto de Corea del Norte. En forma paralela, ambos jefes de gobierno suscribieron una «Agenda de cooperación económica para el siglo 21», lo que dio pie al inicio de las

5 Gutiérrez, H., «Corea en los '90: las estrategias de las economías asiáticas industrializadas ante la globalización», Estudios Internacionales, Año XXXIV, Nº 134, 2001, pp 57-73.

6 Tsutomu Kikuchi, «Japan and Multilaterlism: the regional level», en Regnier, P. y Warner, D. (eds.), Japan and Multilateral Diplomacy, Reino Unido, Ashgate Publishing Limited, 2001, pp. 153-174. 
actuales conversaciones para la firma de un Tratado de Libre Comercio.

\section{Las controversias en torno al factor histórico se circunscriben a círculos} conservadores y nacionalistas.

\section{EL FACTOR HISTÓRICO}

Durante los años noventa, la influencia negativa del factor histórico generalmente no se manifestó de manera pronunciada. Las buenas perspectivas solo se deterioraron a comienzos del 2001, por dos hechos coyunturales que han marcado la agenda posterior entre los dos países. La aprobación por parte del ministerio de educación japonés de una partida de nuevos textos de historia, de marcados tintes nacionalistas, y la visita del primer ministro, Junichiro Koizumi, al Santuario Yasukuni, construido para honrar a los muertos de guerra japoneses. Ambos hechos impactaron negativamente las relaciones bilaterales. Aunque los medios de prensa surcoreanos llamaron incluso a boicotear los productos japoneses, la respuesta popular no fue tan marcada como la registrada en 1982 en circunstancias similares. La explicación, para algunos autores, es simple. La mayoría de los colegios japoneses utiliza otros textos de historia, y no precisamente la edición que dio origen al impasse $^{7}$. Es posible afirmar entonces, que este tipo de controversias se circunscribe a círculos conservadores y nacionalistas que aún cuentan con cierto poder económico que les permite influir en el gobierno y en el público en general ${ }^{8}$. En una visita a Seúl, realizada por el primer ministro Koizumi para apaciguar las críticas, manifestó: «He reflexionado sinceramente sobre el gran daño causado al pueblo coreano durante la ocupación colonial del Japón, y me gustaría ofrecer una disculpa desde lo profundo de mi corazón» ${ }^{9}$. Estas palabras de Koizumi, por un lado, así como sus visitas al santuario Yasukuni, por el otro, parecen reflejar la delicada red de compromisos que existe detrás del gobierno japonés.

Desde un punto de vista constructivista, la persistencia de sentimientos encontrados entre Japón y Corea del Sur es comprensible. Si bien las heridas son muchísimo más profundas del lado coreano, ambas partes han construido su identidad sobre la base de experiencias históricas comunes. El nacionalismo coreano moderno fue precisamente desarrollado por oposición al imperialismo japonés y a la brutal política de asimilación aplicada durante el régimen colonial. En el caso japonés, el mecanismo es más o menos similar, aunque su actitud radica en una persistente autoimagen de superioridad que se remon-

Kim Seong Bo, «History Textbooks of Korea and Japan: A Comparison», Korea Focus, Vol. 10, № 3, 2002. Disponible en <www.koreafocus.or.kr/>.

8 Kim Hosup, «Korea-Japan Relations and Tasks for the Roh Administration», Korea Focus, Vol. 11, $N^{\circ} 3,2003$. Disponible en <www.koreafocus.or.kr/>.

9 Ibidem. 
ta a sus impulsos modernizadores de fines del siglo XIX. Ahora bien, frente al hecho específico de las atrocidades cometidas bajo el régimen colonial, la mayor parte de la población japonesa lo atribuye a una casta militarista, de la cual también habrían sido, indudablemente, víctimas ${ }^{10}$. No obstante estas diferencias, como se desprende de lo vivido en los últimos sesenta años, la dinámica de oposición ha sido paulatinamente reemplazada por una de cooperación, cuestión que quedó reflejada en la organización conjunta de la Copa Mundial de Fútbol del año 2002. Para ambos países, dicha coyuntura representó mucho más que un simple evento deportivo, al punto que el 2002 fue declarado «El año del intercambio nacional Japón-República de Corea». Varios anuncios estuvieron asociados al espíritu de amistad emanado de esta organización conjunta, como el de establecer 2005 como «Año de amistad: Corea-Japón», a fin de conmemorar el $40^{\circ}$ aniversario de la normalización de las relaciones diplomáticas entre ambos países.

\section{LA INFLUENCIA DE Estados Unidos Y CoREA DEL Norte}

La algarabía vivida en Europa por la caída del muro de Berlín y el fin de la Guerra Fría no se extendió del todo al noreste asiático debido a la persistencia del régimen comunista de Kim Il Sung en Corea del Norte. Este hecho influyó decisivamente en la continuación de las alianzas bilaterales de seguridad de Estados Unidos con Japón y Corea del Sur. La continuidad de dichas alianzas también estuvo potenciada por las percepciones de estos dos últimos actores sobre el futuro de la seguridad regional.

\section{La dinámica de oposición se ha reemplazado gradualmente por una de cooperación.}

Desde la perspectiva de Seúl, la alianza de Japón con los Estados Unidos aseguraba la existencia de un obstáculo para una posible evolución remilitarista de Japón; en cambio desde la perspectiva de Tokio, la alianza entre Corea del Sur y los Estados Unidos resultaba conveniente, porque implicaba traspasar los costos de proveer protección militar a la península ante cualquier amenaza surgida desde el norte ${ }^{11}$. De ese modo, la cooperación militar con los Estados Unidos se intensificó desde ambos lados. En forma paralela, tanto Japón como Corea del Sur iniciaron un acercamiento hacia Corea del Norte, inaugurando una dinámica más autónoma aunque complementaria con los Estados Unidos. En un principio, los avances fueron realmente auspiciosos, al punto que en diciembre de 1991 Corea del Norte y

10 Berger, T., «Power and Purpose in Pacific East Asia», en Ikenberry J. y M. Mastanduno (eds.), International Relations Theory and the Asia Pacific, Nueva York, Columbia University Press, 2003, pp. 387-419.

11 Ibidem. 
Corea del Sur firmaron un acuerdo en virtud del cual ambas partes garantizaban la futura desnuclearización de la península. Para Corea del Sur, además, la futura reunificación pacífica con el Norte se transformó en un asunto prioritario de su política exterior.

\section{La reunificación pacífica con Corea del Norte pasó a ser una cuestión prioritaria de su política exterior.}

La positiva evolución del ambiente regional se deterioró a fines de 1992, cuando Japón y Corea del Norte suspendieron las conversaciones destinadas a normalizar sus vínculos diplomáticos. Desacuerdos en torno a la investigación de los secuestros de ciudadanos japoneses ocurridos en la década de los ochenta, por supuestos agentes norcoreanos y demandas de compensaciones de guerra por parte de Corea del Norte hicieron fracasar tal acercamiento. Del mismo modo, la política de renkei planteada por el MOFA sirvió de freno a los avances. Sin embargo, el posterior anuncio por Corea del Norte de que se retiraba del NPT (Tratado de No Proliferación Nuclear), a comienzos de 1993, vino a alterar definitivamente el panorama regional.

La carencia de mecanismos políticos, tanto en Japón como en Corea del Sur, para lidiar directamente con Corea del Norte con el fin de manejar la crisis originada, impulsó a Corea del Sur a recurrir al Consejo de Seguridad de las Naciones Unidas y especialmente a los Estados Unidos para que negociara con el régimen de Kim Il Sung ${ }^{12}$. Después de largas conversaciones, en octubre de 1994 se llegó a un acuerdo, en virtud del cual Corea del Norte aceptó congelar su programa nuclear, permitir las inspecciones del Organismo Internacional de Energía Atómica (OIEA), permanecer dentro del NPT, y reasumir las conversaciones intercoreanas. Los Estados Unidos se comprometieron a proveer de dos reactores nucleares hidroeléctricos a Corea del Norte y a reducir las barreras al comercio y a la inversión en ese país. Para garantizar la construcción de los reactores, se acordó crear la Organización para el Desarrollo Energético de la Península Coreana (KEDO), con la participación de Japón y de Corea del Sur.

La crisis nuclear puso de manifiesto la ausencia de mecanismos efectivos entre los Estados Unidos y Japón para hacer frente a las amenazas regionales a la seguridad. Tal constatación había quedado clara a causa de la falta de coordinación por parte de Japón para el envío de personal y equipo militar a la Guerra del Golfo. Desde el punto de vista estadounidense, era vital que Japón pudiera garantizar ahora apoyo logístico ante cualquier movimiento de sus tropas, incluyendo la participación de las fuerzas de autodefensa japonesas en un eventual bloqueo a Corea del Norte. Pero la respuesta de Japón volvió a ser evasiva. A esto se sumaron 
masivas protestas en las islas de Okinawa sobre la abrumadora presencia de personal militar estadounidense. Aunque la utilidad de la alianza pareció quedar en entredicho, Japón no dudó en revitalizar sus vínculos bilaterales con los Estados Unidos firmando una Declaración Conjunta sobre Seguridad, en abril de 1996.

\section{Para Corea del Sur, la alianza con Estados Unidos se ha convertido en una cuestión de sobrevivencia nacional.}

Para Corea del Sur la alianza con los Estados Unidos se ha convertido en una verdadera cuestión de sobrevivencia nacional, ya que no solo ha disuadido el surgimiento de conflictos en la península; sino que ha permitido la estabilidad del noreste asiático en su conjunto. Para los Estados Unidos, igualmente, la reducción de las tensiones en la península descansa en el establecimiento de un diálogo productivo y de una cooperación más pronunciada entre el Norte y el Sur. Así, inmediatamente después de visitar Japón en abril de 1996, el presidente Clinton propuso, junto al presidente surcoreano Kim Young Sam, la realización de «Conversaciones de Paz de las Cuatro Partes» (Estados Unidos, Corea del Sur, Corea del Norte y China), para reemplazar el armisticio que puso término a la guerra de Corea por un tratado de paz permanente. La iniciativa se concretó un año más tarde, dentro de un contexto de mejoramiento de las relaciones multilaterales.

A mediados de 1997, Japón y Corea del Norte acordaron una vez más reasumir las conversaciones para normalizar sus vínculos diplomáticos. Japón se comprometió a donar 27 millones de dólares en alimentos, mientras que Corea del Norte acordó permitir las visitas a su tierra natal de algunos japoneses que residen en ese país. Al mismo tiempo, se comprometió a investigar los casos de secuestro de ciudadanos japoneses. En diciembre del mismo año se llevó a cabo el primer encuentro entre los países miembros de la ASEAN, por un lado, y Japón, Corea del Sur y China, por el otro, en lo que recibió el nombre de ASEAN+3.

El carácter impredecible de Corea del Norte se puso nuevamente de manifiesto en 1998 con sus pruebas de misiles de largo alcance sobre el espacio aéreo japonés. Tal acción, en medio de la crisis asiática, buscaba ejercer presión sobre los Estados Unidos para que se entablara una nueva negociación respecto del levantamiento de barreras al comercio y a la inversión fijados años atrás ${ }^{13}$. Pero Japón endureció su posición, poniendo fin a las negociaciones con Corea del Norte y rehusando otorgar financiamiento económico para la KEDO. Ante esto, la administración Clinton envió a Pyongyang al ex secretario de defensa, William Perry, para que tratara de llegar a un arreglo amplio que incluyera el tema nuclear y estas políticamente calculadas pruebas de misiles.

13 Harrison, S., «Time to leave Korea?», Foreign Affairs, Vol. 80, N² 2, 2001, p. 63. 
En septiembre de 1999, los Estados Unidos se comprometieron a levantar algunas barreras, a cambio de que Corea del Norte detuviera sus pruebas durante el resto de ese año. En forma paralela, los Estados Unidos, Japón y Corea del Sur establecieron una Coordinación trilateral sobre Corea del Norte, de acuerdo con la cual representantes de los tres países harían una evaluación y seguimiento de sus acciones. A los avances parciales en las relaciones entre Corea del Norte y los Estados Unidos se sumó la sunshine policy del presidente Kim Dae Jung, cuyo primer logro fue una histórica reunión entre ambos líderes en junio del 2000, hecho que presionó a Japón para reanudar las conversaciones con Corea del Norte. Meses más tarde se produjo un intercambio de visitas oficiales entre uno de los hombres de confianza de Kim Jong Il y la secretaria de Estado de los Estados Unidos, Madeleine Albright, que reflejó el mejoramiento de las relaciones bilaterales.

\section{La inclusión de Corea del Norte en el «eje del mal» aumentó los temores de un ataque preventivo estadounidense.}

Sin embargo, el prometedor panorama cambió con la llegada de George W. Bush a la presidencia de los Estados Unidos. El nuevo discurso de la Casa Blanca, que incluyó al régimen de Kim Jong Il en el denominado «eje del mal», aumentó los temores de Corea del Norte de un posible ataque preventivo estadounidense. En consecuencia, el acercamiento entre los Estados Unidos y Corea del Norte se es- tancó, y al mismo tiempo, se detuvieron las relaciones entre Corea del Norte y Corea del Sur. A los pocos meses, mientras la administración Bush comenzaba a preparar su ataque sobre Irak, hizo pública su disposición a reanudar las conversaciones con Pyongyang, pero condicionándolas al cumplimiento de ciertos requisitos. El silencio de Corea del Norte pareció romperse en junio del 2002, con motivo de un incidente registrado en el mar Amarillo entre un barco del Norte con otro del Sur. A pesar de que tal evento no tuvo repercusiones, Washington decidió cancelar una visita del Subsecretario de Estado, James Kelly, que tenía la misión de hacer presente a Kim Jong Il los detalles de una nueva propuesta de acercamiento.

En este ambiente de tensión, Corea del Norte y Japón sostuvieron un histórico encuentro en septiembre del 2002, que allanó el camino para aumentar la ayuda económica del último al primero. Días más tarde, la administración Bush convino en realizar la fracasada visita de James Kelly a Pyongyang, aunque con el objetivo de contrastar informes secretos sobre un posible programa clandestino relacionado con el uso de uranio enriquecido. Semanas después, el Departamento de Estado estadounidense declaró que Corea del Norte había «reconocido» la ejecución de dicho programa, infringiendo el Acuerdo Marco de 1994. De inmediato, hizo un llamado a que Corea del Norte suspendiera el proyecto, subrayando que buscaba una salida pacífica, en cercana consulta con Japón y Corea del Sur. La respuesta de Pyongyang no se hizo esperar y acusó a los Estados Unidos de ser el causante de 
la crisis por no haber cumplido su parte de dicho Acuerdo Marco, específicamente, la construcción de los reactores nucleares hidroeléctricos. Pero la postura de la Casa Blanca se torno inflexible. De los tres países, el que se alineó más rápidamente con Washington fue Japón, que informó a Corea del Norte que la normalización de sus relaciones dependería ahora de la solución del tema nuclear. Corea del Sur, en tanto, enfrentó el problema con mayores dificultades. En medio de elecciones presidenciales, la presencia de las tropas estadounidense se transformó en el centro del debate interno, al punto que el candidato electo, Roh Moo Hyun, llegó a la presidencia en medio de una ola de antiamericanismo ${ }^{14}$.

\section{En Corea del Sur, la presencia de tropas estadounidenses se convirtió en centro del debate interno.}

La seguridad regional empeoró en diciembre de 2002, cuando la KEDO decidió suspender el envío de petróleo a Corea del Norte. En respuesta, Corea del Norte volvió a poner en marcha su infraestructura nuclear, expulsando a los inspectores de la OIEA y anunciando su retiro del NPT en enero del 2003. La administración Bush, impedida de actuar de la misma forma como lo hacía en Irak, continuó buscando ampliar el debate sobre Corea del Norte. En la sesión de junio del 2003 del Foro Regional de ASEAN (ARF), el Secretario de Estado, Colin Powell, aseguró que la crisis nuclear no era un problema bilateral entre los Estados Unidos y Corea del Norte, sino de cada nación que «pudiera caer bajo el arco de un misil norcoreano» ${ }^{15}$. La declaración fue un paso práctico para que las «Conversaciones de las Cuatro Partes» sobre Corea del Norte, se transformaran en las «Conversaciones de las Seis Partes», incluyendo a Rusia y Japón. A septiembre de 2005, este grupo ha celebrado cuatro rondas de encuentros, y aunque no ha arribado a acuerdos concretos, se ha transformado en la única alternativa política hasta el momento para solucionar la presente crisis norcoreana.

\section{LA «AMENAZA» NORCOREANA}

Históricamente, y desde una perspectiva realista, Corea no ha sido nunca una amenaza para nadie. La península siempre ha desempeñando el doble papel de ser una especie de colchón de seguridad para China, en contra de Japón; o una suerte de trampolín continental para este último. Cabe precisar entonces, brevemente, la amenaza objetiva que representa Corea del Norte para el sistema Corea del Sur-Japón, y para su principal elemento coadyuvante, los Estados Unidos

14 Lee Nae-youn, Jeong Han-wool, «Anti-Americanism and the ROK-U.S. Alliance», East Asian Review, Vol. 15, No 4, 2003, pp. 23-46.

15 Chun Min Lee, «Rethinking future paths on Korean Peninsula», The Pacific Review, Vol.17, N 2, 2004, p. 255. 
Pese a que la realidad interna del país concentra, cada vez con mayor intensidad, una yuxtaposición de agudos contrastes, dicha situación no implica que sus capacidades ofensivas se hayan debilitado. Corea del Norte continúa manteniendo un fuerte contingente de fuerzas especiales, con la infraestructura necesaria para desarrollar armas químicas y biológicas, así como misiles balísticos de largo alcance. Aunque sus problemas económicos son patentes, destina entre un tercio y un cuarto de su PIB a los gastos de defensa, sin señales claras de quererlos reducir. Según informes de la CIA, los misiles del tipo Taepodong, de largo alcance, podrían incluso alcanzar las costas de Norteamérica (Alaska), si se logra superar algunos problemas técnicos ${ }^{16}$. En tanto, los misiles Nodong, ya probados, están en condiciones de llegar a Japón, y obviamente, a las bases estadounidenses ubicadas en su territorio. Los vínculos que Corea del Norte ha establecido con Irán y Pakistán en materia de exportación de armamentos son otros de los aspectos que exasperan a los Estados Unidos. De acuerdo con sus analistas estratégicos, el régimen de Kim Jong Il podría incluso vender material nuclear a grupos terroristas «adinerados», tales como $\mathrm{Al}$ Qaeda, con el fin de paliar su crisis económica. Dentro de la escasa y verificable información disponible, es un hecho que el país tiene un reactor nuclear operando en la ciudad de Yangbyon, que puede producir suficiente plutonio para elaborar aproximadamente un arma nuclear por año. Por otro lado, la extrema personalización del poder, primero por parte de Kim Il Sung y luego por su hijo Kim Jong Il, además del carácter totalitario del régimen, dan pie para suponer que un posible colapso interno implicaría un cúmulo de consecuencias regionales inesperadas. Por ejemplo, un conflicto abierto con Corea del Sur, donde un ataque del Norte podría resultar incluso exitoso a corto plazo ${ }^{17}$. Es más, la persistencia de la situación anterior da motivos para que en cualquier momento pueda desencadenarse una escalada armamentista entre los países del noreste asiático en su conjunto.

\section{El colapso del régimen totalitario norcoreano tendría consecuencias regionales inesperadas.}

\section{Conclusiones}

Desde tiempos inmemoriales, la proximidad geográfica ha hecho indudable el establecimiento de una interacción entre Japón y la península coreana y ha determinado la existencia de un subsistema regional dentro del noreste de Asia. Pese a acotados e históricos intentos de aislamiento, como el período Edo japonés, tal posi-

16 Harrison, S., «Time to leave Korea?», Foreign Affairs, Vol. 80, № 2, 2001, p. 65.

17 Pollack, J. y Chung Min Lee, «Preparing for Korean Unification: Scenarios and Implications», pp. 43, 67-73. Disponible en <www.rand.org/publications/MR/MR1040>. 
bilidad se volvió impracticable desde mediados del siglo XIX, cuando ambas partes se vieron obligadas a entrar en contacto con el mundo occidental industrializado. En la actualidad, el grado de modernización de las sociedades, el desarrollo de las tecnologías de la información y la transnacionalización de la economía, hacen ilusorio cualquier ideal de progreso que no contemple a los componentes de cualquier subsistema regional, así como al entorno de ellos.

En términos de seguridad, la evolución histórica de las relaciones entre Japón y la península ha seguido patrones de conflicto y de cooperación, ya sea en la forma de intentos de invasión o de flujos de influencia cultural. El último de los períodos de conflicto, y quizás el más cruento, se vivió desde mediados del siglo XIX a mediados del siglo XX, en la forma del colonialismo japonés. Aunque la fuerza de este legado dejó heridas indelebles en la mayor parte de la población coreana, ha ido paulatinamente disminuyendo debido a la muerte de sus principales protagonistas, pero también por la alteración de la dinámica de interacción entre ambas unidades y a su influencia en la autopercepción de sus respectivas identidades nacionales.

La división de la península, una vez concluida la Segunda Guerra Mundial, dividió también la dinámica de interacción con Japón, perdiendo gran parte de su relativa autonomía y quedando presa de la lógica bipolar de la Guerra Fría. El esquema de equilibrio de poder entre los Estados Unidos y la Unión Soviética se trasplantó así de manera íntegra al noreste asiático, constituyendo la Guerra de Corea un ejemplo práctico de ello. Tanto Japón como Corea del Sur quedaron dentro de la esfera de influencia estadounidense, mediante el establecimiento de alianzas bilaterales de seguridad, mientras que Corea del Norte quedó dentro de la esfera de influencia soviética, o más propiamente, de la de China comunista. De ese modo, Japón se mantuvo en una especie de conflicto técnico con Corea del Norte, mientras que con Corea del Sur se dio paso a una lógica, de acercamiento; que luego se transformo en una lógica de cooperación.

\section{El colonialismo japonés fue el último período de conflicto entre Japón y la península.}

A primera vista, podría afirmarse que después de la Segunda Guerra Mundial Japón alteró dramáticamente su conducta externa mediante la transformación obligada de su estructura política interna. No obstante, tal desarrollo no siguió exactamente el camino de la imposición. Más bien dicho, existían condiciones objetivas que lo posibilitaban, potenciadas por supuesto por la desastrosa derrota en el conflicto. En una palabra, se trató de una cuestión de acentos. Si bien antes la clase política japonesa había explotado el pasado marcial de su historia, el llamado «espíritu del guerrero», ahora, se hacía hincapié en el carácter trabajador y disciplinado de sus ciudadanos, en el respeto por la jerarquía y en la superioridad material del vencedor. Las ideas de paz y de de- 
mocracia occidentales se conjugaron entonces con una nueva forma de apreciar la identidad nacional y la defensa de sus propios intereses, lo que en el fondo permitió que Japón se concentrara más en fortalecer su economía que en su capacidad militar.

\section{Las ideas occidentales de paz y democracia se conjugaron con una forma de apreciar la identidad nacional.}

En Corea del Sur el proceso fue más complejo. Históricamente, la península ha vivido preocupada, respecto de sus vecinos, en ejercer una doble función. Por un lado, la necesidad de afirmar su identidad nacional frente a ellos, pero por el otro, recibiendo una fuerte influencia de estos, e incluso, en ciertos períodos del pasado, buscando directamente su apoyo. Esta conducta ambivalente fue, sin duda, pasto de cultivo para la división ideológica que alcanzó su punto máximo a fines de los años cuarenta y que concluyó con la división territorial del país. Desde ese momento, la actitud posterior de Corea del Sur estuvo principalmente dedicada a contrarrestar el avance de la «amenaza comunista», cayendo en un estado de defensa permanente. La estructura política que de ahí derivó estuvo por tanto acorde con ese propósito, limitando en gran parte el ejercicio de las libertades civiles. Al comienzo, la identidad del país continuó estando determinada exclusivamente por un criterio realista de seguridad nacional, subordinando el peso de la experiencia históri- ca. Pero debido a ese mismo proceso, paradójicamente tendió a incluir a Japón como engranaje clave de su propia defensa. Esta evolución permitiría al poco tiempo echar las bases del posterior y paulatino acercamiento entre ambas partes.

El fin de la Guerra Fría alteró nuevamente la dinámica de interacción entre Corea del Sur, Japón y los Estados Unidos, y en el noreste de Asia en general. La desaparición de la Unión Soviética y la transformación económica de China mermaron las posibilidades de maniobra ofensiva de Corea del Norte, que ha entrado a utilizar su potencial nuclear como una suerte de moneda de cambio para lograr el apoyo a su actual régimen político. En síntesis, la búsqueda de cooperación ha tendido a profundizarse desde diferentes flancos, sobre todo entre Japón y Corea del Sur. Señales claras de este acercamiento entre los distintos países de la región ha sido la llamada tracked diplomacy, que incluye: visitas y encuentros regulares de mandatarios y ministros, declaraciones conjuntas, intercambio cultural y académico, el intercambio económico, y ahora último, el establecimiento de las «Conversaciones de las Seis Partes». El desarrollo particular de este instrumento es un reflejo de las percepciones imperantes en el noreste de Asia. Tras comenzar como una instancia no regular de diálogo que involucraba solo a cuatro países de la región, ha pasado a transformarse en una instancia que intenta buscar una solución pacífica a la crisis nuclear desatada por Corea del Norte en el 2003, con posibilidades serias de proyectarse hacia el futuro como instancia multilateral de seguridad. 
La lógica de cooperación entre Japón y Corea del Sur también se ha dado dentro de un marco en el que ambas unidades comparten principios básicos del liberalismo, tales como la democracia y la economía de mercado. El progreso de sus relaciones ha seguido la evolución política y económica de Corea del Sur desde la época del general Park Chun Hee, en el aspecto económico, y desde el gobierno de Kim Young Sam, en el aspecto político. Sin duda, la sunshine policy del presidente Kim Dae Jung ha marcado uno de los puntos más altos en este progreso. No obstante, la lógica que anima esta evolución, sobre todo en el nivel político, no ha alcanzado plena autonomía, pues sigue dependiendo de las respectivas alianzas que ambos países mantienen con los Estados Unidos, y cuyo desarrollo está asociado directamente a los movimientos de Corea del Norte. Para la mayor parte de los representantes políticos de los países involucrados, estas alianzas han garantizado el mantenimiento de los equilibrios estratégicos en el noreste de Asia, por lo que a mediano plazo no es posible pensar en un retiro de las fuerzas militares estadounidense. Es más, frente al surgimiento de China como potencia mundial, la presencia estadounidense en el área se transforma en un claro mecanismo para equilibrar su poder futuro.

En este nuevo escenario, los Estados Unidos se han visto en la necesidad de reformular su papel estratégico en la región. Una consolidación del subsistema Corea del Sur-Japón, esto es, la constitución de un régimen de seguridad, en los términos planteados por Barry Buzan, permitiría alcanzar tal objetivo, porque se daría dentro de un esquema de cooperación, en el que ninguna de las dos partes consideraría a la otra como una amenaza. Además de ser económicamente beneficioso, es funcionalmente más recomendable en la medida en que los Estados Unidos no se verían obligados a duplicar sus esfuerzos en uno y otro sector, posibilitando una mayor coordinación entre sus fuerzas militares, y entre estas y las fuerzas locales. Una reformulación de estas características también es compatible con el acercamiento entre Rusia y China, que busca precisamente contrarrestar la hegemonía estadounidense en la región.

\section{La división ideológica condujo finalmente a la división territorial del país.}

No cabe duda de que el proceso de reunificación de la península traerá consecuencias para todos los países del noreste asiático, especialmente para China, debido a los potenciales flujos de refugiados. Sin embargo, la incertidumbre mayor gira en torno a la orientación futura que tendría una Corea unificada. Tres son las opciones en este sentido: primero, una Corea más nacionalista; segundo, una Corea más cercana a China y, finalmente, una Corea más pro Estados Unidos. Dado el nivel de compromiso político, económico y de seguridad que existe dentro del subsistema Corea del Sur-Japón, y entre este y los Estados Unidos, las vicisitudes y eventuales complejidades del proceso de reunificación contarían con un mayor apoyo de los dos últimos 


\section{Cristián Maldonado M.}

países. Entre la autonomía y la estabilidad, terior surgimiento de un régimen de seguuna Corea unificada optaría claramente por ridad, esto es, la consolidación de un la segunda, consolidando la cooperación subsistema regional entre la península y triangular en materia de seguridad y el pos- Japón. 